\title{
REVIEW ON MOVING VEHICLE DETECTION IN AERIAL SURVEILLANCE
}

\author{
Ramesh Sejpal ${ }^{1}$,Mayur Charadva ${ }^{1}$, Nisha Sarwade ${ }^{2}$, \\ ${ }^{I}$ M.tech student, Electrical engineer department, Veermata Jijabai Tech. Inst, Maharashtra, India \\ ${ }^{2}$ Professor, Electrical engineer department, Veermata Jijabai Tech. Inst, Maharashtra, India
}

\begin{abstract}
Moving vehicle detection is a key technology for security purpose in urban area. Many researchers got the good results in highway cases but in urban area still improvement in vehicle detection is needed. Cascade classifier method is strongly depending on vehicle size and colour. Morphology is the study of Shape and form of objects. it process the image based on shapes. So it would be the essential in particular method for achieving better detection rate. This paper is intended to present a review of very recent developments to detect moving vehicles. The performance assessment of different methods is evaluated with respect to its detection rate, false position rate and detection speed.
\end{abstract}

Keywords: Aerial surveillance, detection rate, erosion, false positive rate, urban area.

\section{INTRODUCTION}

Florida Department of Transportation has started the project named ATSS (Airborne Traffic Surveillance System) for traffic surveillance to decrease the risk caused by collisionsincidents in highways. Now aerial surveillance also started in urban areas because increment of vehicle users, traffic congestion and incidents in urban scene has become more severe in recent years. So moving vehicle detection is convenient and low-cost technology with easily compatible on an airborne platform during aerial surveillance. Also these technologies have a variety of applications, such as military, police, and traffic management. Here vehicle only include car and bus.

In urban area, there are some additional difficulties than high way cases [1][2][3] like (1) The vehicles move even slower than the camera, which may lead to a lot of false alarm; (2) There are too many moving vehicles in a frame, so two adjacent vehicles may easily be regarded as one and therefore some vehicles may be miss-detected. Coif man, B. et al. [1] proposed a simple but efficient subtraction method for roadway traffic monitoring from an unmanned aerial vehicle.Mirchandani P. et al. [2] proposed a two-frame subtraction method to detect moving vehicles from aerial image and got a high detection rate with low time cost.Yalcin $\mathrm{H}$ et al. [3] proposed an optical flow-based method to detect moving vehicles by segmenting the dense optical flow fields into background and occlusion layers.

These methods could hardly work well in urban traffic surveillance because they cannot solve the above difficulties. For a traditional image subtraction-based method, its performance is greatly determined by the speed of vehicles. If a vehicle moves slowly, the corresponding images in the consecutive frames will have little difference and then the vehicle can hardly be detected by the image subtraction method. Furthermore, objects such as buildings around the road in moving camera platform will lead to a lot of false alarm. Therefore, this method can only be adopted directly for a stationary camera platform.

R.Lin [6] proposed a detection algorithm based on roaddetection. In urban area, most part of area is covered with road so it can be consider as background. By subtracting background vehicle regions would be obtain. After consecutive frame subtraction, moving vehicles detected. X.Cao [7] removes the problem of false positive rate by cascade classifier but detection rate is still low in both discussed methods. At last here proposed the morphological operator [9], if combine with X.cao [7] proposed method then the detection rate would be increase. Morphology is the study of Shape and form of objects. They are useful for object extraction and image segmentation operation.

In section 2, discussed the all-important and related methods briefly. While section 3, compare them with performance parameters. In section 4, here Proposed morphological analysis and at last concluded the paper.

\section{METHODS OF VEHICLE DETECTION}

Image subtraction is the important and base method of all the techniques as subtracts all the pixels from first frame and depending upon the threshold value it detects the vehicles. It might be useful on highway but not useful for urban area where vehicles speed may be slow. Now one by one discuss the other techniques.

\subsection{Image Subtraction with Vehicle Identification}

This method is based upon traditional image subtraction method. Now the advance technique is image subtraction vehicle identification [5], in which vehicle size is added to the traditional image subtraction. It has higher detection rate than traditional method. But it strongly depend upon size of 
vehicles and background of image and uses more area for comparison which are the problems associated with it and decreases the detection rate.

\subsection{Moving Vehicle Detection with Road Detection}

In MVD-RD [6], there is also image subtraction method used but before that Vehicles would be separated from background. Because background cover the most part of area in an image. So the disadvantages of Image subtraction method would be easily overcome as there are less area for comparision.So false alarm rate would be decrease. Here car and bus are considered in vehicle. The Procedure of MVD$\mathrm{RD}$ method is given in fig. 1

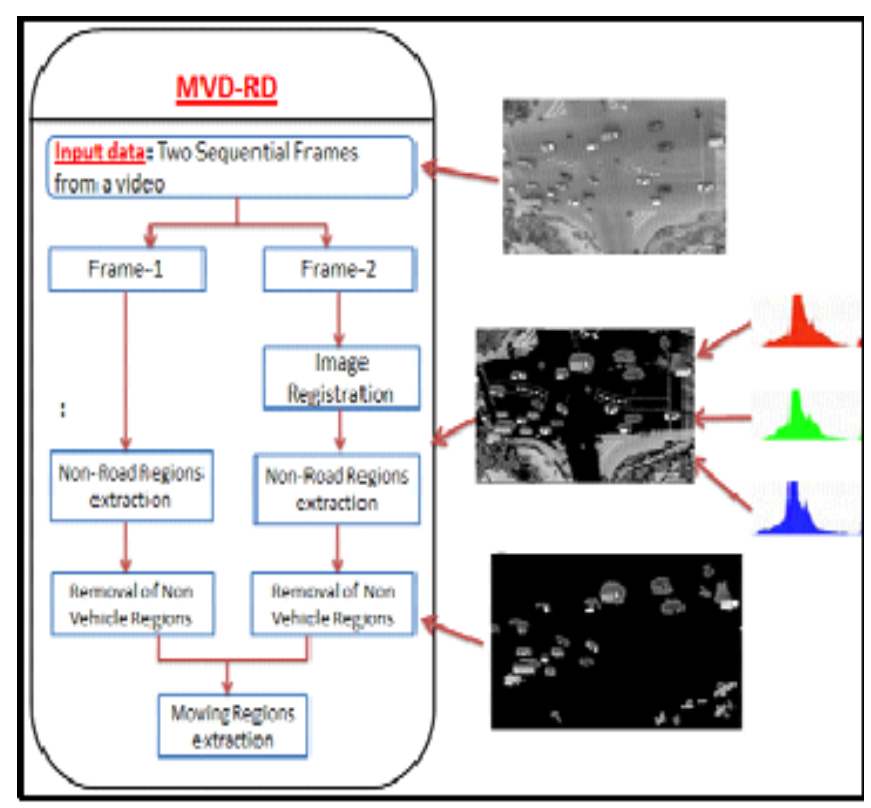

Fig.1 Procedure of MVD-RD

The important steps of MVD-RD method are given below.

1) Take two sequential frames from the video.

2) Frame no. 2 should be translated and rotate with reference to frame-1 because to overcome the effect of camera movement. This method is called Image Registration.

3) For urban area, it is noticeable that road region cover most part of whole frame, so it should be consider as a back ground. By removing that it gets vehicle regions.

4) Remove the regions which are small and larger than vehicles.

5) After that subtracting both the frame it would get the moving regions.

The moving vehicle detection result are shown in fig. 2

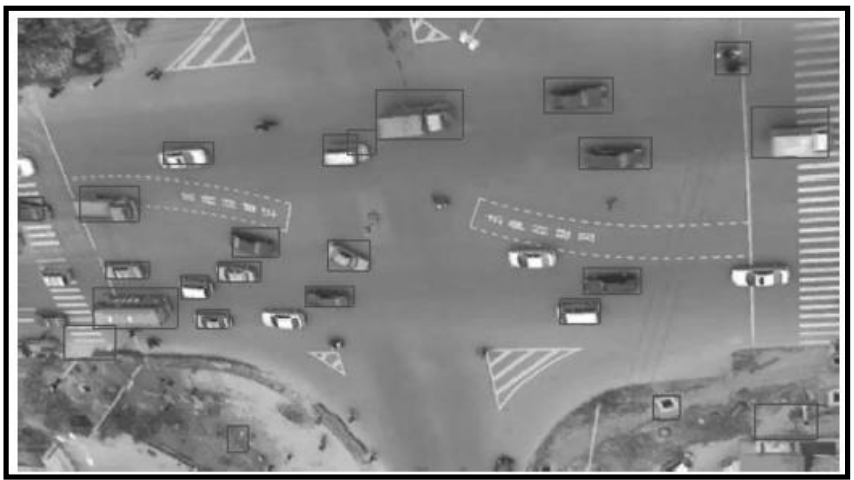

Fig.2 Detected output

\subsection{MVD-RD with Cascade Classifier}

It is the advance method of MVD-RD with including cascade classifier [7].Cascade classifier is made of the feature of vehicles. By using this classifier, false detection is reduced here.The method divide in two part (1) Preprocessing stage (2) Classification stage. Pre-processing stage is the same as MVD-RD. So same procedure is applied here for detecting the moving vehicles and result of preprocessing stage should be the input of Classification stage. Architecture of system is shown in fig.(3).

Requirements for classification stage are:(1) output of preprocessing stage (2) it should train the each classifier with some negative and positive samples, which will useful during identify the each region. So positive samples must be made of vehicles and negative samples must be without vehicles Moreover, The performance of a classifier strongly depends on the features adopted. To extract the efficient feature, Viola and Jones [8] proposed an extremely effective method, and here similar method is used to extract the features.

This method also used MVD-RD so one problem may be solves here to remove false detection but other problem is also pending here that the vehicles which are not detected at output of MVD-RD.

\section{COMPARISON OF ALL METHODS}

The performance of moving vehicle detection depends on
1. Detection rate
2. False positive rate
3. Detection speed

DR and FPR were defined as following:

$$
\mathrm{DR}=\mathrm{T} /(\mathrm{T}+\mathrm{M})
$$

$$
F P R=F /(T+F)
$$

Where $\mathrm{T}$ is average number of detected regions that corresponds to moving vehicles, $\mathrm{F}$ is of detected average number regions that do not correspond to a moving vehicle and $\mathrm{M}$ is average number of moving vehicles not detected. The performance results of all the method are given in Table.1 


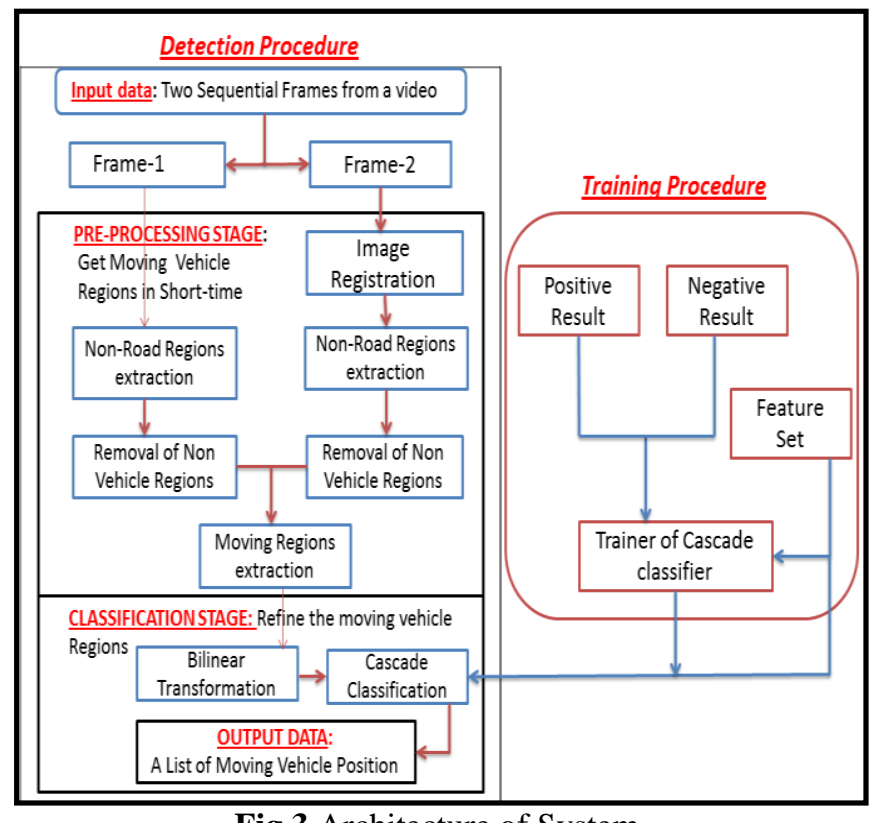

Fig.3 Architecture of System

Table.1 Performance of all the methods

\begin{tabular}{|l|l|l|l|}
\hline $\begin{array}{l}\text { Detection } \\
\text { Method }\end{array}$ & $\begin{array}{l}\text { False } \\
\text { positive } \\
\text { Rate(FPR) }\end{array}$ & $\begin{array}{l}\text { Detection } \\
\text { Speed } \\
\text { (fps) }\end{array}$ & $\begin{array}{l}\text { Detection } \\
\text { Rate(DR) }\end{array}$ \\
\hline $\begin{array}{l}\text { Tradition } \\
\text { Image } \\
\text { Subtraction [1] }\end{array}$ & $>75 \%$ & 8.84 & 75 to $100 \%$ \\
\hline $\begin{array}{l}\text { Image } \\
\text { Subtraction } \\
\text { with vehicle } \\
\text { identification } \\
\text { [5] }\end{array}$ & 25 to $75 \%$ & 8.12 & 70 to $100 \%$ \\
\hline $\begin{array}{l}\text { Moving } \\
\begin{array}{l}\text { Vehicle } \\
\text { detection based } \\
\text { on road } \\
\text { detection [6] }\end{array}\end{array}$ & 0 to $13 \%$ & 6.43 & 80 to $100 \%$ \\
\hline $\begin{array}{l}\text { Cascade } \\
\text { Classifier [7] }\end{array}$ & 0 to 3\% & 6.30 & 80 to $100 \%$ \\
\hline
\end{tabular}

As given in Table.1 Cascade classifier has good Detection rate and low False Positive Rate because of road detection two adjacent vehicles can be separate from each other. Due to feature classification FPR is decreased. One point should mark that both cascade classifier and MVD-RD has same detection rate which should be further improve.

\section{MORPHOLOGICAL ANALYSIS}

Morphology is the study of Shape and form of objects [9]. Morphology image analysis can be used to perform

- Object extraction

- Image filtering operation such as removal of small objects and noise from an image

- Image segmentation operation such as separating connected objects

- Measurement operation such as texture analysis and Shape description
Dilation and erosion are the most basic morphological operations. Dilation is used to shrink the objects from the image. There are also other useful operations such as Opening (erosion followed by dilation) and closing (dilation followed by erosion).Opening is used to remove the small objects from the image. How morphological analysis is useful for the vehicle detection it will be clear from this example. In this example, here taken an image which was used in MVD-RD method and done the morphological erosion. For simulation, MATLAB R2013a software is used here.

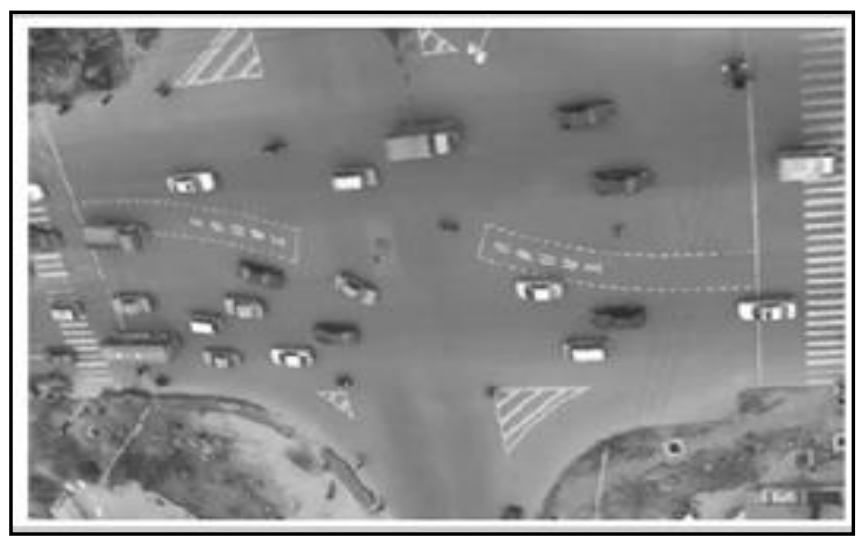

Fig.4 Input image

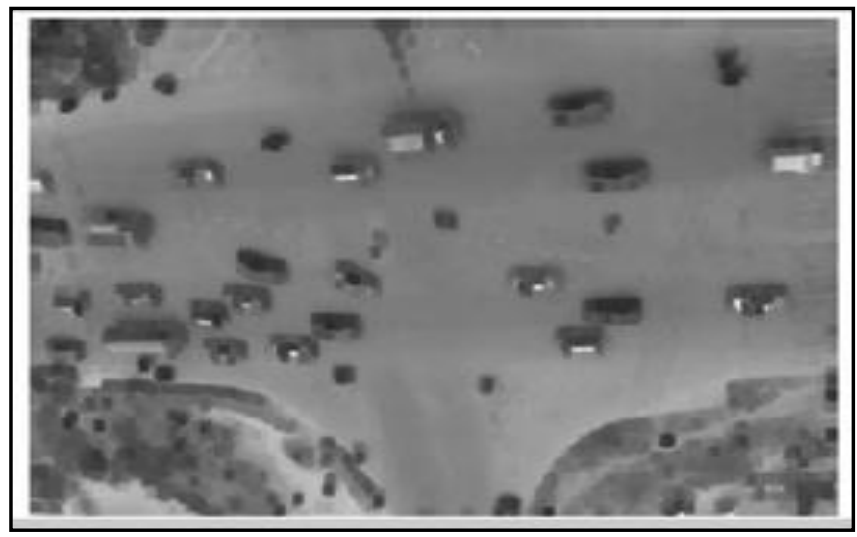

Fig.5 Eroded image

In fig.4 input image is given and after that morphological erosion applied to input image which is given in fig.5. From the figure $4 \& 5$, it is clear that vehicles are highlighted in the image. So if erosion operator is apply before starting road detection then it will be definitely increase the detection rate of MVD-RD method and Cascade classifier method.

\section{CONCLUSIONS}

In the field of moving vehicle detection, many researches have been done and it gradually improved the vehicle detection rate but still this methods need to be improve. Though cascade classifier method has better detection rate $80 \%$ to $100 \%$ but it would work well only when most of the vehicles are dark. So it can be further improve by combining with morphological operators as they figure out the vehicles in the image which would help in removing non vehicle regions. 


\section{REFERENCES}

[1] B. Coif man, M. McCord, R. G. Mishalani, M. Iswalt,"Roadway traffic monitoring from an unmanned aerial vehicle," IEEE Proc. Intell. Transp. Syst vol. 153, no. I, pp: 11-20, 2006

[2] ] P. Sompoch and R. Shibasiki, "Vehicle Detection from Ultra-high Resolution Aerial Image, Three Line Scanner," Geo information Forum Japan, Tokyo Japan, 2003.

[3] H. Yalcin, R. Collins, M. Black, and M. Hebert, "A flow-based Approach to Vehicle Detection and Background Mosaicking in Airborne Video," Proc. IEEE, page1202-1216, 2005.

[4] J. Y. Choi and Y. K. Yang, "Vehicle detection from aerial images using local shape information," $A d v$. Image Video Technol., vol. 5414, Lecture Notes in Computer Science, pp. 227-236, Jan. 2009.

[5] P. Mirchandani, M. Hickman, A. Angle, and D. Chandnani,"Application of Aerial video for Traffic flow parameter and Management" Pecora 15/Land Satellite Information on IV/ISPRS Commission I/FIEOS 2002 Conference Proceedings.

[6] R. Lin, H. Qiao, Y. Xu, C.Wu, X. Cao and, "Airborne moving vehicle detection for urban Traffic surveillance, "in Proc. 11th Int. IEEE Conf. Intell.Transp.Syst,pp. 163-167,Oct. 2008

[7] R. Lin, H. Qiao, Y. Xu, C.Wu, X. Cao, "Airborne moving vehicle detection for video surveillance of urban Traffic" in Proc. IEEE Intell. Veh.Symp., pp. 203-208, 2009

[8] P. Viola and M. jones, "Robust real-time face detection", Int. j. Compute. Vis., vol. 57, no. 2, pp. 137-154, May 2004.

[9] Rafeal C. Gonzalez, "Digital Image Processing", thirdedition,pp. 649-698.

\section{BIOGRAPHIES}

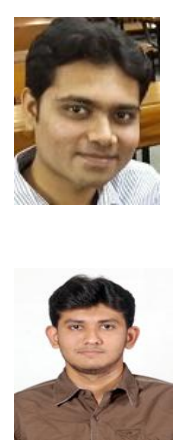

Ramesh Sejpal is a M.tech student from the Veer Mata Jijabai Technological Institute, Mumbai. This paper is a part of his project work.

Mayur Charadva is a M.tech student from the Veer Mata Jijabai Technological Institute, Mumbai. This paper is a part of his project work.

Dr. Nisha Sarwade is a Professor from the Veer Mata Jijabai Technological Institute, Mumbai.This paper is prepared under her guidance She received the Ph.D. degree from IITBOMBAY. 